\title{
The recent trend in mycobacterial strain diversity among extra pulmonary lymph node tuberculosis and their association with drug resistance and the host immunological response in South India
}

Shanmugam Sivakumar ${ }^{1 \dagger}$, Yuvaraj Chandramohan $^{2 \dagger}$, Gokul Raj Kathamuthu $^{3}$, Gomathi Sekar ${ }^{1}$, Devika Kandhasamy', Venkatesan Padmanaban², Syed Hissar ${ }^{4}$, Srikanth P. Tripathy', Ramalingam Bethunaickan², Baskaran Dhanaraj ${ }^{4}$, Subash Babu ${ }^{3}$ and Uma Devi Ranganathan ${ }^{2^{*}}$

\begin{abstract}
Background: Tuberculosis (TB) though primarily affects the lungs it may also affect the other parts of the body and referred as extra pulmonary (EPTB). This study is focused on understanding the genetic diversity and molecular epidemiology of Mycobacterium tuberculosis (M.tb) among tuberculous lymphadenitis (TBL), a form of EPTB patients identified in Chennai, Tamil Nadu.

Methods: The genetic diversity was identified by performing spoligotyping on the M.tb clinical isolates that were recovered from lymph node samples. A total of $71 \mathrm{M}$.tb isolates were recovered from extra pulmonary lymph node samples and subjected to Drug susceptibility testing and spoligotyping was carried out. In addition, immunological characterization from blood of same individuals from whom M.tb was isolated was carried out between the two major lineages groups East African Indian 3 (EAI3) and non-EAI3 strains by ELISA. The results of spoligotyping patterns were compared with the world Spoligotyping Database of Institute Pasteur de Guadeloupe (SpolDB4).

\footnotetext{
* Correspondence: Krumadevi@gmail.com; umadevi.r@nirt.res.in

†Shanmugam Sivakumar and Yuvaraj Chandramohan contributed equally to this work.

${ }^{2}$ Department of Immunology, National Institute for Research in Tuberculosis, Chetpet, Chennai 600 031, India

Full list of author information is available at the end of the article
}

(c) The Author(s). 2020 Open Access This article is licensed under a Creative Commons Attribution 4.0 International License, which permits use, sharing, adaptation, distribution and reproduction in any medium or format, as long as you give appropriate credit to the original author(s) and the source, provide a link to the Creative Commons licence, and indicate if changes were made. The images or other third party material in this article are included in the article's Creative Commons licence, unless indicated otherwise in a credit line to the material. If material is not included in the article's Creative Commons licence and your intended use is not permitted by statutory regulation or exceeds the permitted use, you will need to obtain permission directly from the copyright holder. To view a copy of this licence, visit http://creativecommons.org/licenses/by/4.0/. The Creative Commons Public Domain Dedication waiver (http://creativecommons.org/publicdomain/zero/1.0/) applies to the data made available in this article, unless otherwise stated in a credit line to the data. 
(Continued from previous page)

Results: We found 41 spoligotype patterns and their associated lineages. Out of 41 spoligotype pattern, only 22 patterns are available in the spoldB4 database with Spoligotype international Type (SIT) number and remaining patterns were orphan strains without SIT number. The most predominant spoligotype lineage that was found in lymph node sample in this region of India was EAI (36), followed by central Asian strain (CAS) (6), T1 (5), Beijing (3), Latin American \& Mediterranean (LAM) (2), U (1), X2 (1) and orphan (22). In addition to EAl, CAS and Beijing, our study identified the presence of orphan and unique spoligotyping patterns in Chennai region. We observed six drug resistant isolates. Out of six drug resistant isolates, four were resistant to isoniazid drug and associated with EAl family. Moreover, we observed increased levels of type 2 and type 17 cytokine profiles between EAI3 and nonEAl family, infected individuals.

Conclusions: The study confirms that EAl lineage to be the most predominant lineages in EPTB patients with lymphadenitis and were found to have increased type 1 and type 17 proinflammatory cytokine profiles.

Keywords: Extra pulmonary tuberculosis, Mycobacterium tuberculosis, Spoligotyping, Th1 and Th17 cytokines, Lymph node, Mycobacterial lineage

\section{Background}

Tuberculosis (TB) occurs worldwide and it is caused by an infectious agent Mycobacterium tuberculosis (M.tb). According to World health organization (WHO), there were 1.3 million TB deaths was reported among HIV negative people and an additional 3, 00,000 deaths among HIV-positive people were reported in 2017. TB affected about 10 million people; among them $90 \%$ were adults, 9\% were people living with HIV [1]. The two types of clinical manifestations of $\mathrm{TB}$ are pulmonary (PTB) and Extra-pulmonary (EPTB) tuberculosis. EPTB involves $\mathrm{TB}$ affects body parts other than the lungs, which include lymph nodes, skins, joints, or meninges. Among 6.4 million incident cases, 14\% represents extrapulmonary TB [1]. In India, about 15 to $20 \%$ of EPTB occurs in immune-competent patients and $50 \%$ of cases were reported in HIV-positive individuals [2]. Among them, lymph node TB (35\%), pleural TB (20\%), bone marrow TB (10\%), genitourinary TB (9\%), cerebrospinal, abdominal, skin, etc. accounts for $26 \%$ cases [2]. Though EPTB is not contagious, it has a huge impact on mortality and morbidity rate worldwide. Mycobacterium tuberculosis complex (MTBC) is the most common etiological agent in TB including EPTB and their diagnosis and therapeutics are highly challengeable [3].

Epidemiology of EPTB can be analyzed by genotyping of Mycobacterium tuberculosis, which will be helpful to detect unsuspected transmission, reinfection and relapse and to identify false-positive cultures [4]. The available genotyping methods are IS6110-RFLP, Polymorphic GCrich repetitive sequences (PGRS)-RFLP, Mycobacterial interspersed repetitive unit (MIRU)-VNTR typing and Spoligotyping. Of the available genotyping tools, Spoligotyping and MIRU-VNTR is considered the most suitable tool in clinical settings [5].

Since Spoligotyping (Spacer Oligonucleotide typing) can simultaneous detect and type different lineages of
M. tuberculosis bacterial complex (MTBC). The clinical data obtained by this method had a significant impact on immune surveillance and drug treatment regimens [6]. Previous studies demonstrated that $M$. tuberculosis strains have different epidemiology patterns. Evidence from a variety of sources suggests that MTBC lineages have different phenotypic properties. According to Centers for Disease Control and Prevention (CDC), MTBC genetic lineages are of two categories: Modern lineages and Ancestral lineages. The Modern lineages include East-Asian (Beijing), East-African-Indian (EAI) and Euro-American. The Ancestral lineages include Indo-Oceanic, M. bovis and M. africanum (West African 1 \& West African 2) [7]. Therefore, spoligotypic information on clinical isolates can reveal the phylogenetic and evolutionary relationships among those strains. Previous studies have also documented that EAI (ancient strain) trigger significantly increased expression of certain (TNF- $\alpha$, IL-1 $\beta$, IL-12) cytokines and apoptosis than Beijing (modern lineage) and H37Rv (laboratory strain) up on in exvivo. Also, it has been shown that ancient strains were less lethal when compared to modern strains and they stimulate greater pro-inflammatory host response. In contrast, infection with Mtb Beijing strains might induce non-uniform immune responses [8-11]. In a given situation, characterization of those lineages in EPTB may provide better insights on strain pathogenicity and further will help us to document global tuberculosis epidemiology [12]. Therefore, in this study, we aimed to genotype 71 clinical isolates of $M$. tuberculosis from patients with extrapulmonary tuberculosis (EPTB) to identify the predominant spoligotyping pattern of strains in lymph node samples isolated from Southern India and studied strain specific immune responses among the infected individuals. 


\section{Methods}

\section{Clinical setting}

The strains were received from an ongoing, clinical trial to study 'the efficacy and tolerability of a 4-month regimen containing ofloxacin compared to the standard 6month regimen in the treatment of patients with superficial lymph node TB by National Institute for Research in Tuberculosis (NIRT), Chennai which is a Supranational Reference Laboratory and a WHO collaborating center for TB research and training. From this ongoing study, 71 mycobacterial isolates retrieved from suspected extra pulmonary cases and utilized for performing mycobacterial strain characterization (from the year 2013-2015). The Institutional Ethical Committee (IEC) of National Institute for Research in Tuberculosis (NIRT) approved the study and written informed consent was obtained from the patients before collecting the samples (NIRT IEC 2009 007).

\section{Sample processing, biochemical characterization and identification}

The lymph node samples were collected and transported in Kirchner's medium to the laboratory. Lymph node sample processing: The biopsy sample (Lymph node) is cut into small pieces and grinded with sterile Teflon grinding rod, direct smear is made from the homogenate. The homogenate is treated with $1 \mathrm{ml} 5 \% \mathrm{H}_{2} \mathrm{SO}_{4}$ for $15 \mathrm{~min}$ and neutralized and with sterile distilled water. The deposit is washed and inoculated onto two slopes each of plain Lowenstein Jensen (LJ) and LJ Sodium Pyruvate solid medium, remaining deposit is inoculated into two bottles of SK. LJ medium and SP medium are read for 8 weeks every week before declaring as negative. The SK medium is subculture on to LJ medium if positive. The drug susceptibility testing for First- and second-line anti-TB drugs were carried out for 71 isolates of M.tb. The minimum inhibitory concentration (MIC) was used to predict the sensitive and resistance patterns as per the guidelines recommended by WHO for First- and second-line anti-TB drugs (https://www. who.int/tb/publications/2018/WHO_technical_report_ concentrations_TB_drug_susceptibility/en/). In Brief MIC method was performed using $4 \mathrm{mg}$ moist weight per microlitre (ml) of culture suspension, one-third loopful of 2-3 wk. old culture on L-J medium was suspended in $1 \mathrm{ml}$ of sterile distilled water and vortexed to obtain uniform suspension. The coarse particles or clumps in the suspension were allowed to settle at room temperature. For MIC method, $10 \mu \mathrm{l}$ of the suspension was inoculated onto drug containing and drug free L-J medium. Results were read after 28 days of incubation at $37^{\circ} \mathrm{C}$ for MIC methods. Isolates with $\geq 20$ colony counts ( $1+$ grading) were considered resistant to the particular drug concentration. In addition, MPT-64 Ag detection test were performed for these 71 isolates using SD Bioline TBAg MPT64 Rapid (Standard diagnostics Seoul, South Korea) Kit [13] to confirm M.tb.

\section{DNA extraction}

The DNA extraction from the confirmed $M$. tuberculosis positive patients was carried out as described [14]. The mycobacterial cultures were grown in LJ medium and scrapped in to $1 \mathrm{ml} \mathrm{TE}$ (Tris EDTA) buffer. At $80{ }^{\circ} \mathrm{C}$, the cultures were heat killed for $30 \mathrm{~min}$. To that, $50 \mu \mathrm{l}$ of lysozyme $(10 \mathrm{mg} / \mathrm{ml})$ was added and the isolates were incubated at $37^{\circ} \mathrm{C}$. On the next day, $70 \mu \mathrm{l}$ of $10 \%$ SDS and $6 \mu \mathrm{l}$ of proteinase $\mathrm{K}(50 \mathrm{mg} / \mathrm{ml})$ was added and further incubated at $65^{\circ} \mathrm{C}$ for $15 \mathrm{~min}$. Then, $100 \mu$ lof $5 \mathrm{M} \mathrm{NaCl}$ and $80 \mu \mathrm{l}$ of CTAB were added, incubated again at $65^{\circ} \mathrm{C}$ for $15 \mathrm{~min}$. To the culture, $700 \mu \mathrm{l}$ of chloroform/isoamyl alcohol (24:1) was added and centrifugation was carried out for $20 \mathrm{~min}$ at $10,000 \mathrm{~g}$ at $25^{\circ} \mathrm{C}$. The supernatant was collected into a new Eppendorf tube. To precipitate the DNA, $600 \mu \mathrm{l}$ of ice-cold iso-propanol was added to the tubes and incubated at $-20^{\circ} \mathrm{C}$ overnight. On the third day, Eppendorf tubes were subjected to centrifugation at $12,000 \mathrm{rpm}$ for $15 \mathrm{~min}$ and the pellet was washed with $500 \mu \mathrm{l}$ of $70 \%$ ethanol. The extracted genomic DNA was re-suspended in TE buffer and stored at $20^{\circ} \mathrm{C}$ until use. To confirm the presence of DNA, the extracted DNA was run on $1 \%$ agarose gel electrophoresis and quantified using Nano drop.

\section{Spoligotyping}

Spoligotyping was performed as described previously [15]. DRa and DRb primers were used for amplifying the direct repeat (DR) region in the genome of MTB complex. M. tuberculosis H37Rv strain and Mycobacterium bovis BCG P3 chromosomal DNA were used as positive controls. The molecular biology grade water served as negative control. The commercial membrane pre-coated with spacer-oligos, which represents the spacer region of known sequences, was used for hybridizing the amplified product. The membrane was incubated with streptavidin-peroxide and ECL to visualize the presence/ absence of spacer on X-ray film as black squares. Based on the combination of the presence/absence of theses spacers in a M.tb strain is distinguished as spoligotype. The available spoligotyping patterns in international spoligotype database (SpoIDB4) were used for comparing the spoligotyping patterns of lymph node samples obtained from this study and SIT number is assigned.

\section{Elisa}

In order to study the host immune response, plasma samples obtained from EPTB infected with respective strains were used to measure the systemic levels of different (IFN $\gamma$, TNF $\alpha$, IL-2, IL-4, IL-5, IL-13, IL-12, IL17A, IL-22, IL-10, IL-1 $\alpha$, IL-1 $\beta$, IL-6 and GM-CSF) 
cytokines and CC/CXC chemokines (CCL-1/I-309, CCL2/MCP-1, CCL3/MIP1 $\alpha$, CCL4/MIP-1 $\beta$ and CCL 11/eotaxin, CXCL-1/Gro- $\alpha$, CXCL2/ Gro- $\beta$, CXCL9/ MIG, CXCL10/IP-10 and CXCL11/ITAC 1) by bio-plex ELISA. The experiment was carried out according to the manufactures instruction provided ( $R$ \& D systems).

\section{Data analysis}

The immunological parameters were analyzed using GraphPad PRISM (GraphPad Software, Inc.,San Diego, CA, USA). Geometric means (GM) were used for measurements of central tendency. Finally, Mann-Whitney $\mathrm{U}$ test was used to find the statistically significant differences among the cytokines and chemokines.

\section{Results}

We recovered 71 Mycobacterium tuberculosis strains from lymph node collected from EPTB patients recruited under this study. Majority of the patients recruited in this study were aged more than 18 years and other demographic and clinical details were reported in supplementary Table 1.

\section{Drug resistance and EPTB recurrence}

Out of the 71 isolates tested, 65 isolates were sensitive to all first line and second line anti-TB drugs. Four isolates were resistant to isoniazid; one isolate was mono resistant to rifampicin and one isolate shows resistant to ofloxacin (Table 1).

\section{Spoligotyping}

The isolated cultures from the lymph node were subjected to spoligotyping and it resulted in 41 spoligotype patterns as per the SpolDB4 database [16]. The resulted 41-spoligotype patterns and their associated lineages are shown in Fig. 1. The phylogenetic tree for the spoligotype pattern was generated using unweighted pair group method and arithmetic mean (Fig. 2). Out of 41 spoligotype pattern, only 22 patterns are available in the spoldB4 database with Spoligo international Type (SIT) number and remaining patterns were identified as orphan strains without SIT number. The most predominant spoligotype lineage that was found in lymph node sample in this region of India was EAI (36), followed by CAS (6), T1 (5), Beijing (3), LAM (2), U (1), X2 (1) and orphan (22). We looked at the age and gender association, though the numbers $(n=71)$ are few to draw any conclusion, we noticed females were more $(74.6 \%)$ represented compared to males (25.4\%). Majority of the common strains are EAI3_IND and Orphans ND and strains are represented by all age group (Supplementary Fig. 2A \& B).
Table 1 Shows the number of different lineages identified by SpoldB4 database

\begin{tabular}{|c|c|c|}
\hline S. NO & SPOLIGOTYPES & $\begin{array}{l}\text { NO OF ISOLATES } \\
\text { (NO OF RESISTANT STRAINS) }\end{array}$ \\
\hline 1. & Beijing 1 & $3(c-1)$ \\
\hline 2. & CAS 1345 & 1 \\
\hline 3. & CAS 142 & 1 \\
\hline 4. & CAS 601 & 1 \\
\hline 5. & CAS1_DELHI 26 & 3 \\
\hline 6. & EAl 3 Orphan & $9(a-3)$ \\
\hline 7. & EAl 5 Orphan & 9 \\
\hline 8. & EAl1_SOM 270 & 1 \\
\hline 9. & EAl1_SOM 48 & $3(d-2)$ \\
\hline 10. & EAl1_SOM 734 & 1 \\
\hline 11. & EAI3_IND 11 & 20 \\
\hline 12. & EAl3_IND 473 & 1 \\
\hline 13. & EAI5 126 & 2 \\
\hline 14. & EAI5 340 & 1 \\
\hline 15. & EAl5 355 & 1 \\
\hline 16. & EAI5 474 & 1 \\
\hline 17. & EAI5 517 & 1 \\
\hline 18. & EAI5 733 & 1 \\
\hline 19. & EAI5 938 & 2 \\
\hline 20. & EAl6_BGD1 591 & $1(a-1)$ \\
\hline 21. & Harleem1 Orphan & 3 \\
\hline 22. & LAM9 42 & 2 \\
\hline 23. & Orphan & 22 \\
\hline 24. & S Orphan & 1 \\
\hline 25. & T1 53 & $5(b-1)$ \\
\hline 26. & U 172 & 1 \\
\hline 27. & X2 137 & 1 \\
\hline
\end{tabular}

a-Isoniazid resistant; b-Rifampicin resistant; c-Ofloxacin resistant; $d$-Recurrence

\section{Sub-lineages identified from lymph node samples}

Among the EAI family, the most predominant lineage was EAI3 (21 Nos) followed by EA15 (9 Nos), EAI1 (5 Nos) and EAI6 (1 Nos). The sub-lineages identified from lymph node samples are summarized in Table 1. A total of 21 isolates belongs to EAI3, comprising 20 isolates in EAI3_IND and 1 isolate in EAI3_IND 473. The next predominant lineage of EAI family is EAI5 (9 No) which are further classified in to different sub lineages as follows: EAI5 126 (2 Nos), EAI5 340 (1 No), EAI5 355 (1 No), EAI5 474 (1 No), EAI5 517 (1 No), EAI5 733 (1 No) AND EAI5 938 (2 Nos). The third predominant lineage of EAI family is EAI1 ( $5 \mathrm{Nos}$ ) and was further identified to belong to four sub-lineages EAI1_SOM 270 (1 No), EAI1_SOM 48 (3 Nos) andEAI1_SOM 734 (1 No) EAI6 (1 No). Total of 18 new variants of EAI were found without SIT numbers. The next prevalent lineage among 71 isolates of lymph node sample is CAS family (6 Nos). A total of 6 isolates belongs to CAS, comprising 3 isolates 


\section{Spoligotype distribution}

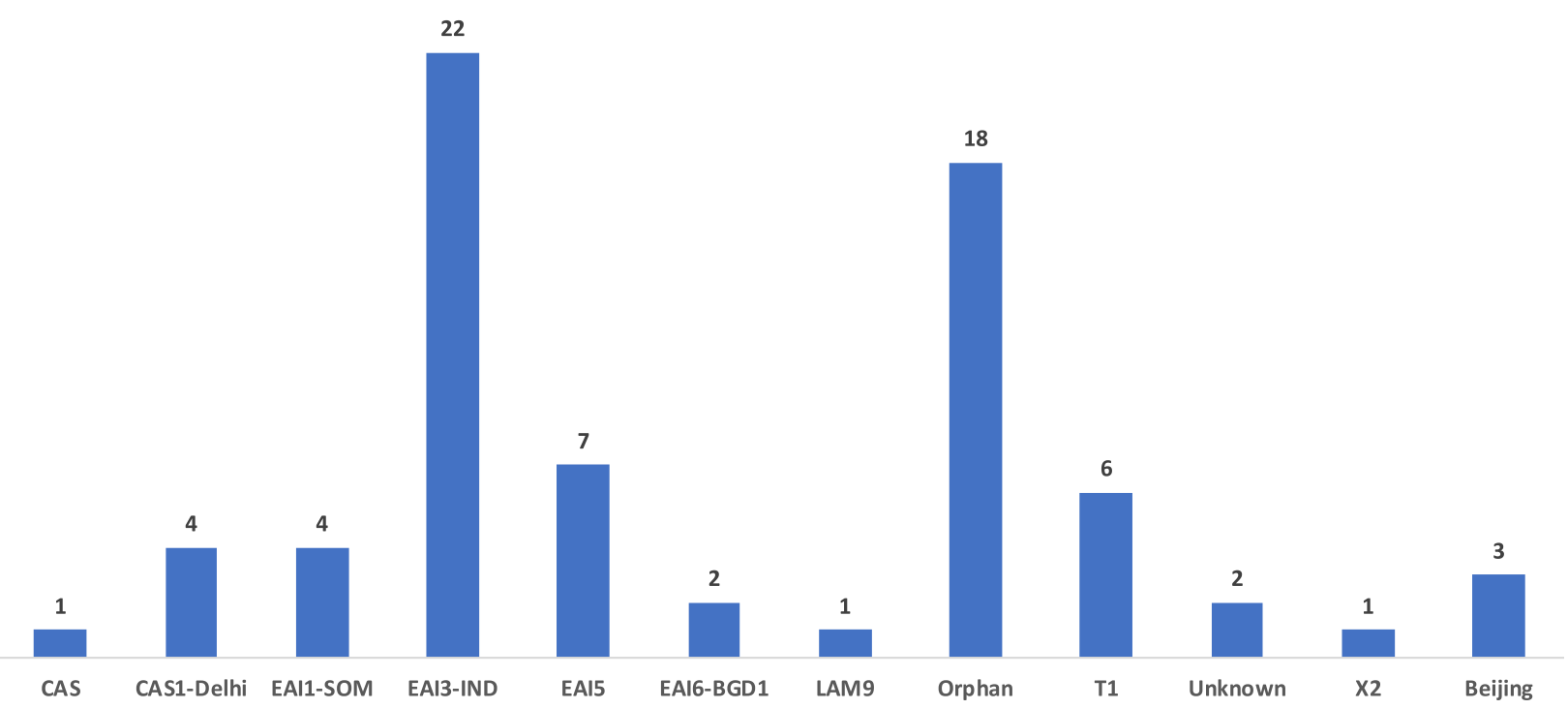

Fig. 1 Spoligotype patterns and their associated lineages of 71 isolates from lymph node samples

in CAS_DELHI 26 followed by CAS 1345 (1 No), CAS 142 ( $1 \mathrm{No}$ ) and CAS 601 (1 No). The existing spoldB4 database was compared and the patterns, which do not match the existing information, are called orphan strains. A total of 22 orphan strains were identified from this study.

\section{Spoligotyping pattern and their associated drug resistance}

Out of 71 isolates tested, 65 were sensitive to all the first- and second-line anti-TB drugs and belong to different lineages like EAI, CAS, LAM, U and X2. The spoligotype patterns and their associated drug resistance were analyzed in this study. We observed six drug resistant isolates. Out of six drug resistant isolates, four were resistant to isoniazid drug and associated with EAI family. Mostly, the sub-lineages of EAI3 orphan (3 Nos) and EAI6_BGD1 (1 No) are associated with H mono resistance (Table 1). The remaining two strains were mono resistant to rifampicin and ofloxacin and belong to $\mathrm{T} 1$ and Beijing lineage respectively. In addition, we found recurrence of the disease in two patients out of 71 patients tested, they were primarily infected with EAI1_SOM sub-lineage, which is different from the most prevalent circulating EAI3 strains in south India (Table 1).

\section{Differential immune response among patients infected with EAI3 and non - EAI 3 strains}

To determine whether any significant immunological differences are associated with the individuals infected with either EAI3 India or non-EAI3 strains, we have measured the different (type 1, type 2, type 17, IL-1 family and pro-inflammatory) cytokines using the available plasma samples of respective EPTB individuals (Supplementary Fig. 1). Among them, the plasma levels of IL-2 and IL- $1 \alpha$ were significantly higher in individuals infected with non-EAI3 India strains upon comparison with EAI3 India strains. In contrast, IL-13 cytokine level was significantly reduced in non-EAI3 India strain infected individuals when compared to EAI3 India strains (Fig. 3a). Similar to cytokines, we have also examined the systemic levels of different CC chemokines between the EAI3 India and non-EAI strains. As shown in Fig. $3 \mathrm{~b}$, the CC (CCL-3/ MIP- $1 \alpha$ and CCL-4/ MIP-1 $\beta$ ) chemokines were significantly different (diminished in EAI3 India strain infected individuals) between the two study groups.

\section{Discussion}

A major public health concern, which still remains and contributes to the world epidemiology, is tuberculosis. For development of effective infection control and vaccines, the frequency of various M.tb genotype and their transmission patterns need to be analyzed using molecular tools such as spoligotyping and MIRU-VNTR. To differentiate recurrence, re-infection and mixed infections in multiple strains of $M . t b$ these genotyping methods are widely used $[17,18]$. Previous reports from our group have already reported the circulating lineages of south India from pulmonary TB samples [19]. However, this was the first report from our group, which focuses on 


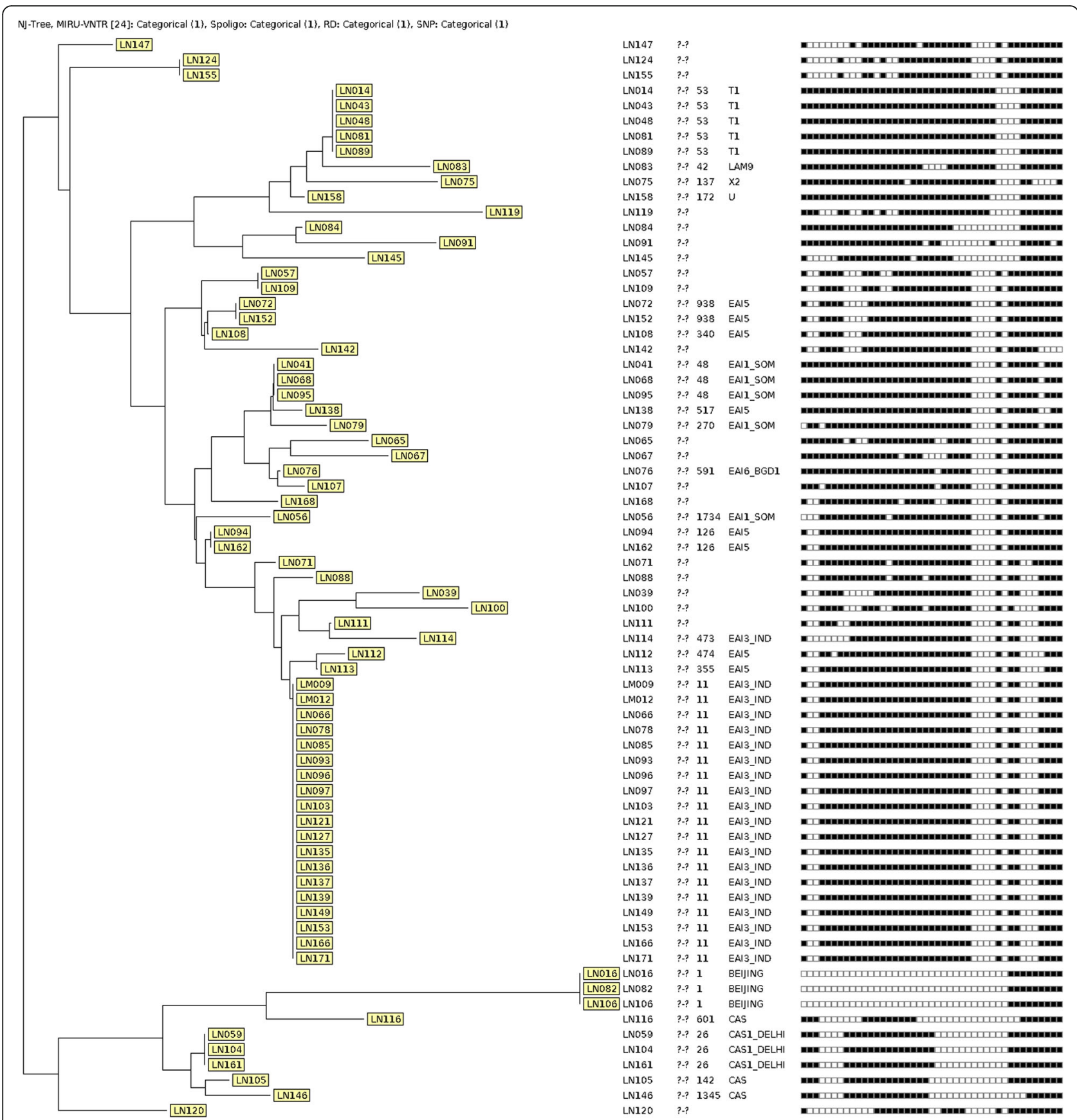

Fig. 2 Phylogenetic tree of 71 isolates from lymph node samples

circulating lineage of extra pulmonary lymph node samples from ongoing clinical trials. In this study, we mainly focus on circulating lineages of extrapulmonary lymph node samples and their associated drug resistance pattern. Not many molecular epidemiological studies have been performed for lymph node extra pulmonary sample. In this context, this study provides a great insight and a better understanding of circulating lineages and their associated drug resistance patterns of extra pulmonary lymph node samples.

We performed Spoligotyping and Immunological assays for the extrapulmonary lymph node samples to understand the genetic diversity of different circulating strains that were recovered from ongoing clinical trial. Our present study identified $76.05 \%$ of EAI strains from Chennai, which was isolated from lymph nodes samples, 


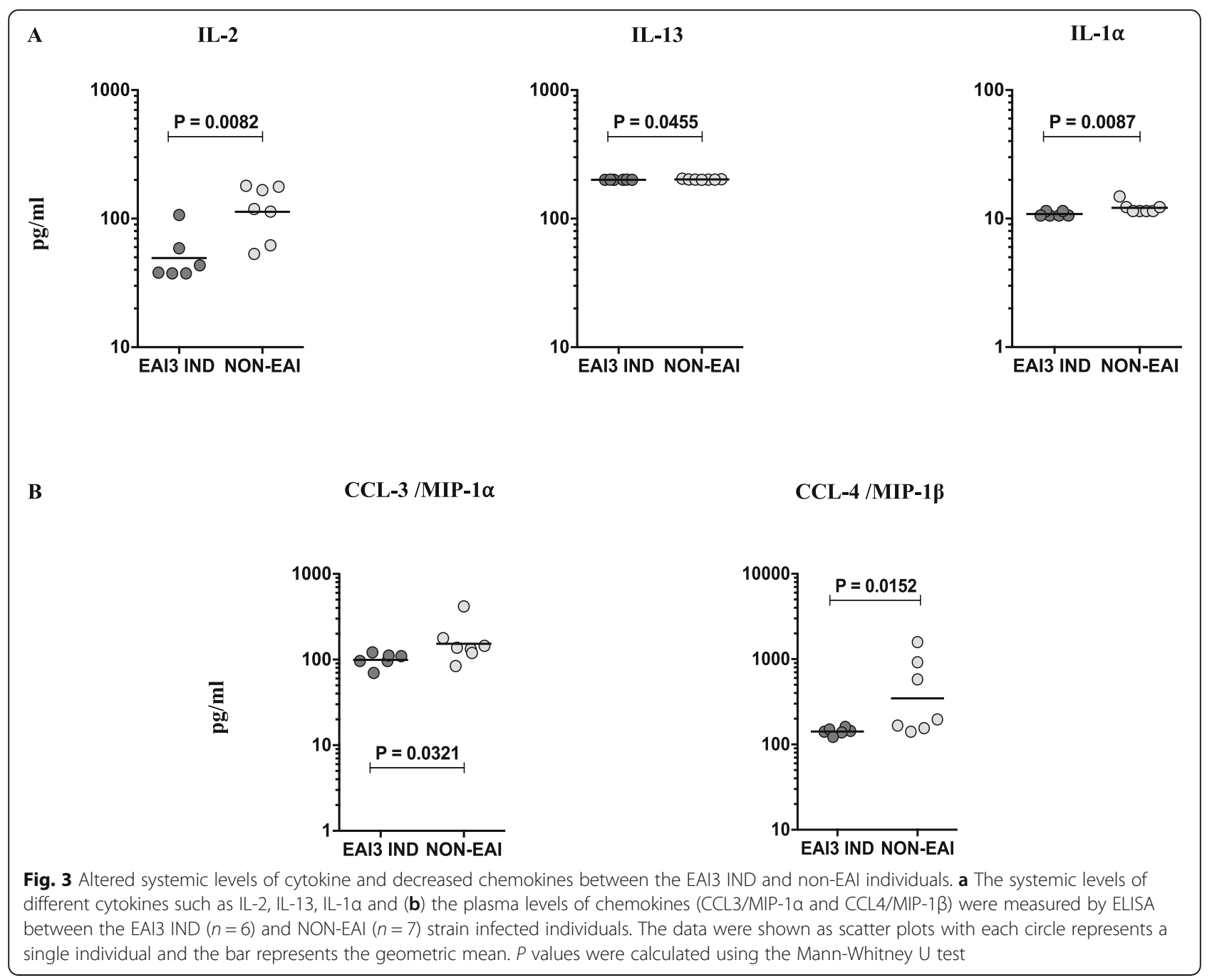

and found to be most predominant strains circulating in south India. This is in line with the previous studies, which identified 67.5 and $59.3 \%$ of EAI strains from pulmonary M.tb isolates $[19,20]$. In comparison with North India, the high prevalence of EAI strains in pulmonary MTB isolates has already been reported in Kerala and Chennai [19-22]. The highest percentage of EAI strains (89\%) were previously reported in Kerala [20]. Similarly, Joseph and co-workers also reported $64.3 \%$ of EAI strains in 2013 [21]. Various reports from North India mentioned 3.8\% EAI strains from Delhi [18], 4.44\% EAI strains from Maharashtra [23], 9.6\% in UP and Varanasi [24] and 10\% from the whole of north India [3]. Singh et al., 2007 reported that EAI lineages are more predominant in south India, whereas the CAS family is predominating the northern parts of India [25]. The origin, evolution and migration of two populations in India and the differences in lineages shows the presumption that Mycobacterium tuberculosis strains might have originated and evolved in a different way [3]. Similar study showed that Euro-American lineage were three times more likely to generate a secondary case during the 11-year study period than patients infected with any other strain. The data clearly shows that the immune response and genetic background plays a major role in deciding the infection with a particular lineage of $\mathrm{Myco}$ bacterium tuberculosis [26]. In the present study, we identified one EAI6_BGD1 from lymph node samples. A similar finding has been reported for the first time in Puducherry [27]. In the current study, drug susceptibility testing was carried out for all the isolates of lymph node samples. Majority of the strains were susceptible to first line drugs and six isolates were resistant to drugs like rifampicin, isoniazid and ofloxacin. Out of six isolates tested, all the four isolates are from EAI family and were resistant to Isoniazid. These resistant strains belong to EAI3 orphan strains (3 Isolates) and EAI6_BGD1 (1 Isolate). 
The central and Middle Eastern Asia (CAS) family were the second largest Clade identified in our study; this is reported to be the predominant lineage in North India previously. Our study identifies $7.89 \%$ of CAS strain from lymph node samples, which accounts for the low prevalence of CAS family in Chennai. This is similar to the findings of previous research workers who also recorded the low prevalence of CAS family in south India, which accounts $1.5 \%$ in Trivandrum [20], 3.5\% in Chennai [19] and $3.5 \%$ in Kerala [21]. Contrary to this low prevalence of CAS family in south India, North India recorded a very high prevalence of CAS family (57.2\%) from extra pulmonary samples [3]. Similarly, Singh et al., 2007 reported 56.5\%, 53.6 and 44.6\% from Delhi, Pune and Lucknow, respectively [20]. This evidence clearly suggests that there are contrasting lineages circulating in north and south India. It is well-established historical fact that North Indians belongs to the Aryan descent whereas, South Indians belongs to Dravidian culture. This might be contributing for the demarcation of prevalent strains in different parts of India. In contrast, a study by Thomas et al., reported the higher prevalence of CAS family in Andhra Pradesh. This could be due to the migration of people to Hyderabad from Middle East countries and north India which contributes for the increased prevalence of CAS family.

The prevalence for Beijing strains in different parts of India ranges from $2.2 \%$ [19] to $11.5 \%$ [20] for pulmonary specimens. In the case of extra pulmonary specimens, the prevalence was slightly higher with the percentage of 10 and $18.8 \%[3,28]$. The Beijing strain was found to be more prevalent in multi drug extrapulmonary TB specimen and treatment failure cases with the percentage of $63.6 \%[23,28]$. In our study, Beijing strain was identified at a rate of $3.94 \%$ from lymph node. One of our lymph node samples were found to be resistant to Ofloxacin and associated with Beijing family. Previous studies from rural areas of Hyderabad and Mumbai have shown that Beijing strains were absent. However, in urban population of Andhra Pradesh it was prevalent at a rate of 6.8 and $10.4 \%$ among treatment failure cases [23, 29]. In addition to Beijing, the high rate of drug resistance is also associated with Haarlem lineages. This study reports the prevalence of Haarlem strain at a rate of $3.94 \%$ but none of these lineages contributed for the drug resistance genotypes. Interestingly, outside India the frequency of Beijing strains was more (56\%) in TB meningitis patients which was reported by two consecutive studies from Bangkok and Thailand [30, 31].

In our study among the orphans, we found 22 unique patterns which were missing in SpolDB4, 9 M.tb Strains show features of EAI3 strains and 9 show similarity to EAI5 and three to Haarlem and one to $\mathrm{S}$ spoligotype. Previous reports by Desikan and his colleagues from
Madhya Pradesh reported three EPTB isolates were found to be orphan [32]. In addition to this we also found very rare genotypes like LAM (2.63\%), X2 (1.31\%), $\mathrm{U}(1.31 \%)$ and $\mathrm{S}(1.31 \%)$ lineages in our study. This is in line with a study by Sankar et al. [3] who reports the rare isolates of LAM and X2 from extra pulmonary specimens. Furthermore, our study also identified Five T1 isolates from extra pulmonary specimens and for the first time one such report is also reported by Kandhakumari et al. [27] in India. In addition, drug susceptibility testing also found among six isolates tested; only one isolates belonging to the T1 lineage was found resistant to one of the first line drug rifampicin. We had four relapse in our study and genotyping results was available for two of them and both belong to EAI1_SOM. Recurrent infections have been reported earlier by us in HIV infected TB patients by EAI1_SOM M.tb strains [33]. Further studies need to carry out to explore association of EAI1_SOM M.tb strains in recurrence.

Furthermore, exogenous reinfections were observed by many researchers in both immunocompetent and immunosuppressed individuals. Mostly, drug resistant strains and exogenous reinfections from drug sensitive organisms have been reported in so many lineages. Two patients in our study had a recurrent TB infection. Moreover, immunological characterization studies were performed between EAI and Non-EAI family in order to understand the differences in cytokine profiles which confers drug resistance to this particular lineage. Immune protection against tuberculosis are mediated by the induction of $\mathrm{T}$ cell responses specifically through type 1 and type 17 cytokines [34]. Besides, IL-1 family (IL-1 $\alpha$, IL-1 $\beta$ and IL-18) and IL-12 cytokine are also necessary for protection against Mtb [35]. However, the relationship or the association of strain specific immune mediated responses in EPTB individuals has not been examined so far. Hence, for the first time we show there was significant immunological differences were observed between the individuals infected with EAI3 India and non-EAI strains. The major differences observed are for IL-2, IL-1 $\alpha$ (reduced in EAI3 India strain) and IL-13 (increased in EAI3 strain). Decreased IL-2 levels in the EAI3 India strain infected individuals suggest that the former is associated with loss of immune protection and persistent chronic infection associated in EPTB individuals. The complete loss of IL-2 eventually leads to reduced immunity and expansion of Mtb growth and increased IL-2 provides protection along with substantial expansion of central memory T-cells $[36,37]$. Like IL-2, IL-1 family cytokines are also equally important for immune protection and our data shows diminished levels of IL- $\alpha$ in EAI3 India strain infected individuals than the non-EAI. Previous studies also shown that deficiency in the IL-1 family cytokines are more susceptible to TB 
infection [35]. Finally, Type 2 cytokine (IL-13) were significantly enhanced in individuals infected with EAI3 India strain. Type 2 cytokines were harmful to host immune response to $\mathrm{TB}$ disease [34] and increased levels associated with chronic infection among the EAI3 India strain than non-EAI strain infected individuals. Therefore, individuals with EAI3 infected strains might have suppressed host protective (Type 1) responses mediated by Type 2 cytokines. Apart from those, type 1 (IFN $\gamma$, TNF $\alpha$ ), type 2 (IL-4, IL-5), type 17 (IL-17, IL-22), regulatory (IL-10) and pro-inflammatory (IL-6, IL-12 and GMCSF) cytokines were not significantly different between the two strains (data were not shown).

In addition, we have also analyzed some of the major CC and CXC (CCL-1/I-309, CCL2/MCP-1, CCL3/MIP1 $\alpha$, CCL4/MIP-1 $\beta$ and CCL11/eotaxin, CXCL-1/Gro- $\alpha$, CXCL2/ Gro- $\beta$, CXCL9/MIG, CXCL10/IP-10 and CXCL11/ITAC 1) chemokines between the EAI3 India and non-EAI strain infected individuals. Interestingly we found CCL3/MIP1 $\alpha$ and CCL4/MIP-1 $\beta$ were higher in non-EAI strain infected individuals (Supplementary Fig. 1). Both chemokines have an important role in migration, homing, recruitment and stimulation of leukocytes to the sites of inflammation [38]. Hence the immune system fails to protect individuals infected with EAI3 strains against ЕРTB infection. Further, we have also examined these cytokine and chemokine response to in drug sensitive (DS) versus Ofloxacin mono resistant (OMR) strains. Similar results were also observed between the two groups where increased Type 2 (IL-4 and IL-13) and Type 17 (IL17 and IL-22) cytokines were observed in OMR strain infected individuals (Supplementary Fig. 1). It has been shown that Type 17 cytokine plays a crucial function in controlling Mtb through recruitment of immune cells, effective granuloma formation at early stages of infection. Meanwhile increased production might have the detrimental effects by promoting immunopathology [39]. Similar to EAI3 and non-EAI3 groups, the other cytokines and chemokines do not possess any significant differences between the DS and OMR (data were not shown) strains infected individuals. These observations reinforce that cytokines or chemokines involved in the host protection are compromised or suppressed in the EAI3 India strain or in OMR resistant strain infected individuals. Using only spoligotyping as the method for molecular epidemiology was limitation of our study, but we wanted to classify our strains into broader range, so that we can better study the immune response developed by the different spoligotypes. The other limitation is small sample number and drug resistance strains in EPTB samples analyzed.

\section{Conclusion}

The present study provides molecular epidemiological data of mycobacteria in lymph node samples isolated from extra-pulmonary patients from south India. The study confirms that EAI lineage to be the most predominant lineages in EPTB patients with lymphadenitis and were found to have increased type 1 and type 17 proinflammatory cytokine profiles. The presence of orphan and unique spoligotyping patterns in this study identifies the genetic diversity of $M . t b$ strains in Chennai region and more molecular epidemiological studies are recommended in lymph node samples throughout India to elucidate their role in TB lymphadenitis.

\section{Supplementary information}

The online version contains supplementary material available at https://doi. org/10.1186/s12879-020-05597-0.

Additional file 1: Supplementary Table 1. Demographic and clinical details of the study population

Additional file 2: Supplementary Fig. 1. Diminished plasma levels type 2 and type 17 cytokines associated with individuals infected with drug sensitive strain. The systemic levels of IL-4, IL-13, IL-17A and IL-22 were measured by ELISA between the drug sensitive (DS, $n=10$ ) and orphan mono resistant $(\mathrm{OMR}, n=3)$ strain infected individuals. The data were shown as bar graph and $P$ values were calculated using the MannWhitney $U$ test.

Additional file 3: Supplementary fig. 2A. The M.tb spoligotypes distribution among male and female patients. Supplementary fig. $\mathbf{2 B}$. The M.tb spoligotypes distribution among different age groups.

\section{Abbreviations}

M.tb: Mycobacterium tuberculosis; MTBC: Mycobacterium tuberculosis bacterial complex; EAl: East African Indian; CAS: Central Asian Strain; LAM: Latin America and Mediterranean; PGRS: Polymorphic GC-rich repetitive sequences; MIRU: Mycobacterial interspersed repetitive unit; SIT: Spoligotype international type; OMR: Ofloxacin mono resistant; DS: Drug sensitive; EPTB: Extra pulmonary tuberculosis; MIC: Minimum inhibitory concentration; IL: Interleukin; CCL: Chemokine (C-C motif) ligand; MCP: Monocyte chemoattractant protein; MIP: Macrophage inflammatory protein; CXCL: Chemokine (C-X-C motif) ligand; MIG: Monokine induced by gamma interferon; IP-10: IFNY inducible protein 10; ITAC 1: Interferon-inducible T cell alpha chemoattractant 1

\section{Acknowledgments \\ The authors thank the staff of the Department of Clinical Research, NIRT, Government Stanley Hospital, Government General Hospital and \\ Government Kilpauk Medical Hospital, Chennai for valuable assistance in recruiting the samples for this study.}

\section{Authors' contributions}

Conceived and designed the experiments: SS, YC, UDR. Performed the experiments: SS, YC, GRK. Analyzed the data: SS, YC, GRK. Contributed materials/reagents/analysis tools: GS, DK, VP, SH, SPT, RB, DB, SB, UDR. Wrote the paper: GRK, SS, YC, UDR. The authors have read and approved the final manuscript.

\section{Funding}

This work was supported by Indian Council of Medical Research (ICMR)Intramural funding of National Institute for Research in Tuberculosis (NIRT).

\section{Availability of data and materials}

The data and materials supporting this article are included within the article.

\section{Ethics approval and consent to participate}

The Institutional Ethical Committee (IEC) of National Institute for Research in Tuberculosis (NIRT) approved the study and written informed consent was obtained from the patients before collecting the samples (NIRT IEC 2009 007). 


\section{Consent for publication}

Not applicable.

\section{Competing interests}

None declared.

\section{Author details}

'Department of Bacteriology, National Institute for Research in Tuberculosis, Chetpet, Chennai 600 031, India. ${ }^{2}$ Department of Immunology, National Institute for Research in Tuberculosis, Chetpet, Chennai 600 031, India. ${ }^{3}$ National Institute of Health -International Center for Excellence in Research National Institute for Research in Tuberculosis, Chennai, India. ${ }^{4}$ Department of Clinical Health Research, National Institute for Research in Tuberculosis, Chetpet, Chennai 600 031, India. ${ }^{5}$ National Institute for Research in Tuberculosis, Chetpet, Chennai 600 031, India.

Received: 19 May 2020 Accepted: 8 November 2020

Published online: 26 November 2020

\section{References}

1. WHO Global Tuberculosis Report, 2018.

2. Sharma SK, Ryan H, Khaparde S, Sachdeva KS, Singh AD, et al. Index- TB guidelines: guidelines on extrapulmonary tuberculosis for India. Indian J Med Res. 2017;145(4):448-63.

3. Sankar MM, Singh J, Diana SC, Singh S. Molecular characterization of mycobacterium tuberculosis isolates from north Indian patients with extrapulmonary tuberculosis. Tuberculosis (Edinb). 2013;93:75-83.

4. Pang Y, Zhou Y, Zhao B, Liu G, Jiang G, Xie H, et al. Spoligotyping and drug resistance analysis of mycobacterium tuberculosis strains from national survey in China. PLoS One. 2012;7(3):e32976.

5. Kato-Maeda M, Metcalfe JZ, Flores L. Genotyping of mycobacterium tuberculosis: application in epidemiologic studies. Future Microbiol. 2011; 6(2):203-16.

6. Gori A, Bandera A, Marchetti G, Esposti A, Catozzi L, Nardi GP, et al. Spoligotyping and mycobacterium tuberculosis. Emerg Infect Dis. 2005. 11(8):1242-8.

7. Aminian M, Shabbeer $A$, Bennett KP. Determination of major lineages of mycobacterium tuberculosis complex using mycobacterial interspersed repetitive units. Proceedings IEEE Int Conf Bioinformatics Biomed. 2009;2009: 338-43.

8. Mitchison DA, Selkon JB, Lloyd J. Virulence in the Guinea pig, susceptibility to hydrogen peroxide, and catalase activity of isoniazide sensitive tubercle bacillifrom south Indian and British patients. J Pathol Bacteriol. 1963;86:37786.

9. Portevin D, Gagneux S, Comas I, Young D. Human macrophage responses to clinical isolates from the mycobacterium tuberculosis complex discriminate between ancient and modern lineages. PLoS Pathog. 2011;7: e1001307.

10. Aguilar D, Hanekom M, Mata D, Gey van Pittius NC, van Helden PD, et al. Mycobacterium tuberculosis strains with the Beijing genotype demonstrate variability in virulence associated with transmission. Tuberculosis (Edinb). 2010;90:319-25.

11. Dormans J, Burger M, Aguilar D, Hernandez-Pando R, Kremer K, et al. Correlation of virulence, lung pathology, bacterial load and delayed type hypersensitivity responses after infection with different mycobacterium tuberculosis genotypes in a BALB/C mouse model. Clin Exp Immunol. 2004; 137:460-8.

12. Nebenzahl-Guimaraes H, Sultana R, lartchouk O, Bozeman S, Galagan J, Sisk $P$, et al. Genetic determinants of drug resistance in mycobacterium tuberculosis and their diagnostic value. Am J Respir Crit Care Med. 2016; 194(5):621-30

13. Orikiriza P, Nyehangane D, Atwine D, Kisakye JJ, Kassaza K, Amumpaire JM, et al. Evaluation of the SD bioline TB Ag MPT64 test for identification of mycobacterium tuberculosis complex from liquid cultures in southwestern Uganda. Afr J Lab Med. 2017;6(2):1-4

14. Wood J. Molecular cloning. In: A laboratory manual by T Maniatis, E F Fritsch and J Sambrook. New York: Cold Spring Harbor Laboratory; 1982 p. 545. Biochem Educ. 1983;11(2):82. https://doi.org/10.1016/03074412(83)90068-7.

15. Kamerbeek J, Schouls L, Kolk A, van Agterveld M, van Soolingen D, et al. Simultaneous detection and strain differentiation of mycobacterium tuberculosis for diagnosis and epidemiology. J Clin Microbiol. 1997;35:90714.

16. Brudey K, Driscoll JR, Rigouts L, Prodinger WM, Gori A, Al-Hajoj SA, et al. Mycobacterium tuberculosis complex genetic diversity: mining the fourth international spoligotyping database (SpolDB4) for classification, population genetics and epidemiology. BMC Microbiol. 2006;6(1):23.

17. Lazzarini LC, Rosenfeld J, Huard RC, Hill V, Lapae Silva JR, De Salle R, et al. Mycobacterium tuberculosis spoligotypes that may derive from mixed strain infections are revealed by a novel computational approach. Infect Genet Evol. 2012:12(4):798-806.

18. Mallard K, McNerney R, Crampin AC, Houben R, Ndlovu R, Munthali L, et al. Molecular detection of mixed infections of mycobacterium tuberculosis strains in sputum samples from patients in Karonga District, Malawi. J Clin Microbiol. 2010;48(12):4512-8.

19. Shanmugam S, Selvakumar N, Narayanan S. Drug resistance among different genotypes of mycobacterium tuberculosis isolated from patients from Tiruvallur, South India. Infect Genet Evol. 2011;11:980-6.

20. Singh UB, Arora J, Suresh N, Pant H, Rana T, Sola C, et al. Genetic biodiversity of mycobacterium tuberculosis isolates from patients with pulmonary tuberculosis in India. Infect Genet Evol. 2007;7:441-8.

21. Joseph BV, Soman S, Radhakrishnan I, Hill V, Dhanasooraj D, Kumar RA, et al. Molecular epidemiology of mycobacterium tuberculosis isolates from Kerala, India using IS6110-RFLP, spoligotyping and MIRU-VNTRs. Infect Genet Evol. 2013;16:157-64

22. Singh UB, Suresh N, Bhanu NV, Arora J, Pant H, Sinha S, et al. Predominant tuberculosis spoligotypes, Delhi, India. Emerg Infect Dis. 2004;10:1138-42.

23. Chatterjee A, D'Souza D, Vira T, Bamne A, Ambe GT, Nicol MP, et al. Strains of mycobacterium tuberculosis from western Maharashtra, India, exhibit a high degree of diversity and strain-specific associations with drug resistance, cavitary disease, and treatment failure. J Clin Microbiol. 2010;48:3593-9.

24. Mathuria JP, Sharma P, Prakash P, Samaria JK, Katoch VM, Anupurba S. Role of spoligotyping and IS6110-RFLP in assessing genetic diversity of mycobacterium tuberculosis in India. Infect Genet Evol. 2008;8:346-51.

25. Singh UB, Arora J, Suresh N, Pant H, Rana T, Sola C, et al. Genetic biodiversity of mycobacterium tuberculosis isolates from patients with pulmonary tuberculosis in India. Infect Genet Evol. 2007:7(4):441-8.

26. Gagneux S, DeRiemer K, Van T, Kato-Maeda M, de Jong BC, Narayanan S, et al. Variable host-pathogen compatibility in mycobacterium tuberculosis. Proc Natl Acad Sci U S A. 2006;103(8):2869-73.

27. Kandhakumari G, Stephen S, Sivakumar S, Narayanan S. Spoligotype patterns of mycobacterium tuberculosis isolated from extra pulmonary tuberculosis patients in Puducherry, India. Indian J Med Microbiol. 2015;33:267-70.

28. Vadwai V, Shetty A, Supply P, Rodrigues C. Evaluation of 24-locus MIRUVNTR in extrapulmonary specimens: study from a tertiary Centre in Mumbai. Tuberculosis (Edinb). 2012:92:264-72.

29. Thomas SK, Iravatham CC, Moni BH, Kumar A, Archana BV, Majid M, et al. Modern and ancestral genotypes of mycobacterium tuberculosis from Andhra Pradesh, India. PLoS One. 2011;6:e27584

30. Faksri K, Drobniewski F, Nikolayevskyy V, Brown T, Prammananan T, Palittapongarnpim $\mathrm{P}$, et al. Epidemiological trends and clinical comparisons of mycobacterium tuberculosis lineages in Thai TB meningitis. Tubercul. 2011;91:594-600.

31. Yorsangsukkamol J, Chaiprasert A, Prammananan T, Palittapongarnpim P, Limsoontarakul S, Prayoonwiwat N. Molecular analysis of mycobacterium tuberculosis from tuberculous meningitis patients in Thailand. Tubercul. 2009:89:304309.

32. Desikan P, Chauhan DS, Sharma P, Panwalkar N, Gautam S, Katoch VM. A pilot study to determine genetic polymorphism in mycobacterium tuberculosis isolates in Central India. Indian J Med Microbiol. 2012;30:470-3.

33. Narayanan S, Swaminathan S, Supply P, Shanmugam S, Narendran G, Hari L, et al. Impact of HIV infection on the recurrence of tuberculosis in South India. JID. 2010;201(5):691-703.

34. O'Garra A, Redford PS, McNab FW, Bloom Cl, Wilkinson RJ, Berry MP. The immune response in tuberculosis. Annu Rev Immunol. 2013;31:475-527.

35. Mayer-Barber KD, Andrade BB, Barber DL, Hieny S, Feng CG, Caspar P, et al. Innate and adaptive interferons suppress IL-1a and IL-1 $\beta$ production by distinct pulmonary myeloid subsets during mycobacterium tuberculosis infection. Immunity. 2011;35:1023-34.

36. Toossi Z, Kleinhenz ME, Ellner JJ. Defective interleukin 2 production and responsiveness in human pulmonary tuberculosis. J Exp Med. 1986:163. $1162-72$. 
37. Millington KA, Innes JA, Hackforth S, Hinks TS, Deeks JJ, Dosanjh DP, et al. Dynamic relationship between IFN-gamma and IL-2 profile of mycobacterium tuberculosis-specific T cells and antigen load. J Immunol. 2007;178:5217-26.

38. Moser B, Wolf M, Walz A, Loetscher P. Chemokines: multiple levels of leukocyte migration control. Trends Immunol. 2004;25:75-84.

39. Khader SA, Bell GK, Pearl JE, Fountain JJ, Rangel-Moreno J, Cilley, et al. IL 23 and IL-17 in the establishment of protective pulmonary $\mathrm{CD4}^{+} \mathrm{T}$ cell responses after vaccination and during mycobacterium tuberculosis challenge. Nat Immunol. 2007;8:369-77.

\section{Publisher's Note}

Springer Nature remains neutral with regard to jurisdictional claims in published maps and institutional affiliations.

Ready to submit your research? Choose BMC and benefit from:

- fast, convenient online submission

- thorough peer review by experienced researchers in your field

- rapid publication on acceptance

- support for research data, including large and complex data types

- gold Open Access which fosters wider collaboration and increased citations

- maximum visibility for your research: over $100 \mathrm{M}$ website views per year

At $\mathrm{BMC}$, research is always in progress.

Learn more biomedcentral.com/submissions 